\title{
Comparison of Compliance of Adjuvant Chemotherapy Between Laparoscopic and Open Surgery in Patients With Colon Cancer
}

\author{
Kan Ho Chun, Byung Noe Bae, Hoon An, Hyeonseok Jeong, Hyunjin Cho, Geumhee Gwak, Keun Ho Yang, \\ Ki Hwan Kim, Hong Ju Kim, Young Duk Kim \\ Department of Surgery, Inje University Sanggye Paik Hospital, Seoul, Korea
}

Purpose: Many studies have shown that the completion of adjuvant chemotherapy improves the survival rate. Recently, laparoscopic surgery has been used to treat patients with colon cancer. We analyzed the relationship between the completion of adjuvant chemotherapy and the operation method.

Methods: We retrospectively analyzed the medical records of 147 patients diagnosed with colon cancer from January 1, 2009, to May 31, 2012. The numbers of patients who underwent laparoscopic and open surgery were 91 and 56, respectively. We analyzed the relationship between the operation method and various factors such as the completion rate of chemotherapy, the patient's age, gender, and physical activity, the postoperative hospital stay, the start time of chemotherapy, and the patient's body mass index (BMI), TNM stage, and type of health insurance.

Results: In the laparoscopic surgery group, the postoperative hospital stay $(13.5 \pm 14.82$ days vs. $19.6 \pm 11.38$ days, $\mathrm{P}=0.001)$ and start time of chemotherapy ( $17.7 \pm 17.48$ days vs. $23.0 \pm 15.00$ days, $\mathrm{P}=0.044)$ were shorter, but the percent complete of chemotherapy (71/91 [78.0\%] vs. 38/56 [67.8\%], $\mathrm{P}=0.121)$, and survival rate (88/91 [96.7\%], 47/56 [83.9\%], $\mathrm{P}=0.007)$ were higher than they were in the open surgery group. Patients who were elderly, had a low BMI, and a high American Society of Anesthesiologists score were less likely to complete adjuvant chemotherapy than other patients were.

Conclusion: Laparoscopic surgery shows a shorter postoperative hospital stay, a shorter start time of chemotherapy, and a higher survival rate. Laparoscopic surgery may be expected to increase compliance of chemotherapy and to improve survival rate.

Keywords: Adjuvant chemotherapy; Colon neoplasms; Percent complete; Laparoscopy

\section{INTRODUCTION}

According to 2011 statistical data from the National Cancer Center, a total of 28,112 colon cancer cases occurred, and its crude incidence rate was 39 per 100,000 population. These data show that colon cancer is the third most common cancer diagnosed in Ko-

Received: Aug 21, 2014 - Accepted: Oct 29, 2014

Correspondence to: Byung Noe Bae, M.D.

Department of Surgery, Inje University Sanggye Paik Hospital, 1342 Dongil-ro, Nowon-gu, Seoul 139-707, Korea

Tel: +82-2-950-1023, Fax: +82-2-3391-4393

E-mail: bnbae@paik.ac.kr

(C) 2014 The Korean Society of Coloproctology

This is an open-access article distributed under the terms of the Creative Commons Attribution NonCommercial License (http://creativecommons.org/licenses/by-nc/3.0) which permits unrestricted noncommercial use, distribution, and reproduction in any medium, provided the original work is properly cited. rea. Also, colon cancer accounts for $11.1 \%$ of all cancer deaths and represents the fourth highest mortality rate. The colon cancer five-year survival rate has been gradually increasing over the last 15 years, with a $54.8 \%$ increase from 1993 to 1995 and a $73.8 \%$ increase from 2007 to 2011 [1]. Such improvements in the survival rate are thought to be mainly due to advances in adjuvant chemotherapy and surgical techniques [2]. Large-scale clinical studies on laparoscopic surgery for treating patients with colon cancer have consistently shown that patients who undergo laparoscopic surgery have some advantages, including shorter hospital stay and recovery period, early postoperative ambulation and physical activity, and reduced postoperative pain [3-5]. We have hypothesized that these advantages might help patients receive their postoperative adjuvant chemotherapy on schedule, so we investigated whether or not the type of surgical procedure had any effect on the compliance with adjuvant chemotherapy in patients who un- 
derwent colon cancer surgery. Moreover, we hypothesized that various clinical and pathological factors might also have an effect on the compliance with adjuvant chemotherapy. Therefore, we analyzed the dependences of the differences in the timing to initiate adjuvant chemotherapy and in its completion rate on clinical and pathological variables, and we investigated the survival rate.

\section{METHODS}

We retrospectively analyzed the medical records of 147 patients who were diagnosed with colon cancer and underwent surgery at Sanggye Paik Hospital between July 1, 2009, and May 31, 2012. In this study, there were no strict criteria to determine the preferable surgery method between laparoscopic and open surgery. However, in 2009, laparoscopic surgery was mostly offered to patients with tumors equal to or less than $5 \mathrm{~cm}$ in size or with body mass indices (BMIs) less than 23. However, since 2012, it has been offered to patients who underwent a colonic stent placement for large bowel obstruction, but enough decompression had to have been achieved before the surgery. Of patients diagnosed with stage II or III colon cancer on a postoperative biopsy test, the patients who were thought to be at high risk based on the American Joint Committee on Cancer (AJCC), 7th edition TNM classification received adjuvant chemotherapy. With the patient's consent, the adjuvant chemotherapy was initiated when the patient was able to eat solid food and it did not disturb the bowel movement. A total of 147 patients were classified into two groups: 91 patients underwent laparoscopic surgery, and 56 patients underwent open surgery.

We investigated the length of a hospital stay after the surgery, the timing to initiate chemotherapy, and the percent complete of chemotherapy to evaluate the compliance of adjuvant chemotherapy according to the surgery method. In addition, we analyzed the relationships between factors such as gender, age, BMI, physical activity levels, and the completion rate of chemotherapy in order to identify the correlations with the biological characteristics of the patients. The 2013 registration data on overall survival rates of subjects were provided by the National Cancer Center upon our request. The length of a postoperative hospital stay was defined as the number of days from surgery (day 0 ) until discharge from the hospital. The timing to initiate chemotherapy was defined as the number of days from surgery (day 0) until the first day of adjuvant chemotherapy. Based on BMI values, underweight patients (20 $\mathrm{kg} / \mathrm{m}^{2}$ or less) were assigned to the first group, and normal-weight or overweight patients than $20 \mathrm{~kg} / \mathrm{m}^{2}$ ) were assigned to the second group. Large bowel obstruction was defined as the presence of air or fluid levels on plain abdominal radiographs taken 3 days after the surgery. The physical status of each patient was evaluated based on the American Society of Anesthesiologists (ASA) score suggested by the ASA and was used to assess the fitness of each patient before surgery. Healthy patients and subjects with mild systemic disease were assigned 1 point and 2 points, respectively, and they were assigned to the first group. Patients with severe systemic disease and patients with severe systemic disease that was a constant threat to life were assigned 3 and 4 points, respectively, and they were assigned to the second group. No patient was assigned 5 or 6 points based on the ASA score.

Oxaliplatin in combination with 5-fluorouracil (Folfox), Oxaliplatin in combination with Capecitabin (Xelox), Tegafur+Uracil and Capecitabine were used for adjuvant chemotherapy. If patients received 8 cycles of Folfox, 6 cycles of Xelox, and 6 months of oral chemotherapy, the chemotherapy was regarded as complete. This study used the chi-square test and the student t-test for statistical analysis. If the P-value was equal to or less than 0.05 , the results were considered statistically significant.

\section{RESULTS}

Of 147 patients with colon cancer, 91 and 56 patients underwent laparoscopic and open surgery, respectively. Their average age was $70.0 \pm 10.06$ and $69.0 \pm 13.00$ years, respectively $(\mathrm{P}=0.450)$. The gender ratio was $57: 34$ and $41: 15$, respectively $(\mathrm{P}=0.127)$. As a result of classifying the patients based on the AJCC 7th edition TNM classification, the numbers of patients with stages II and III colon cancer in the laparoscopic group were 48 and 43 , respectively, and the numbers in the open surgery groups were 35 and $21(\mathrm{P}=$ 0.162 ). The numbers of patients covered by Koreass National Health Insurance and medical aid program were 83 and 8 in the laparoscopic group and 50 and 6 in the open surgery group, respectively. No statistically significant differences in the patients' characteristics were noted between the two groups $(\mathrm{P}=0.454)$ (Table 1).

The average lengths of stay in the hospital after the surgery were 13.5 and 19.6 days in the laparoscopic and the open surgery group, respectively. The length of stay in the hospital was shorter in laparoscopic group and this result was statistically significant $(\mathrm{P}=0.001)$. The chemotherapy was started, on average, 17.7 and 23.0 days after the surgery in the laparoscopic and the open surgery group, re-

Table 1. Patients' characteristics according to the operation method

\begin{tabular}{lccc}
\hline Characteristic & LS & OS & P-value \\
\hline Age (yr), mean \pm SD & $70.0 \pm 10.06$ & $69.0 \pm 13.00$ & 0.450 \\
Gender & & & 0.127 \\
$\quad$ Male:female & $57 / 34$ & $41 / 15$ & \\
$\quad$ BMl (kg/m²), mean \pm SD & $23.5 \pm 3.84$ & $23.6 \pm 3.45$ & 0.361 \\
ASA score & & & 0.258 \\
$\quad$ 1,2/3,4 & $77 / 13$ & $44 / 11$ & \\
Stage & & & 0.162 \\
$\quad$ I/III & $48 / 43$ & $35 / 21$ & \\
Health insurance & & & 0.454 \\
NHI/medicaid & $83 / 8$ & $50 / 6$ & \\
\hline
\end{tabular}

LS, laparoscopic surgery; OS, open surgery; SD, standard deviation; BMI, body mass index; ASA, American Society of Anesthesiologists; NHI, National Health Insurance. 
Table 2. Compliance of chemotherapy according to the operation method

\begin{tabular}{lccc}
\hline Variable & LS & OS & P-value \\
\hline Hospital stay (day) & $13.5 \pm 7.27$ & $19.6 \pm 11.38$ & 0.001 \\
Starting day of chemotherapy (day) & $17.7 \pm 17.48$ & $23.0 \pm 15.00$ & 0.044 \\
Completion rate of chemotherapy & $71 / 91(78.0)$ & $38 / 56(67.8)$ & 0.121 \\
Overall survival rate & $88 / 91(96.7)$ & $47 / 56(83.9)$ & 0.007 \\
\hline
\end{tabular}

Values are presented as mean \pm standard deviation or number (\%). LS, laparoscopic surgery; OS, open surgery.

spectively. This result showed that the laparoscopic group started their chemotherapy earlier, and this difference between the two groups was statistically significant $(\mathrm{P}=0.044)$. Of the 91 patients who received laparoscopic surgery, 71 patients $(78 \%)$ completed their adjuvant chemotherapy. In addition, of the 56 patients who received open surgery, 38 patients $(67.8 \%)$ completed their adjuvant chemotherapy. The completion rate was higher in the laparoscopic group, but this difference was not statistically significant $(\mathrm{P}=0.121)$. Based on the 2013 registration data provided by the National Cancer Center upon our request, the overall survival rates of patients were $96.7 \%$ ( 88 of 91) and $85.7 \%$ (48 of 56) in the laparoscopic and the open surgery group, respectively $(\mathrm{P}=0.007)$ (Table 2).

As a result of analyzing the causes of prolonged hospital stay after surgery, surgical wound infection was more common in the open surgery group, and this result was statistically significant $(\mathrm{P}$ $=0.043$ ). Other possible causes of prolonged hospital stay, such as bowel obstruction, urinary tract complications, including dysuria, hematuria, and urinary tract infections, and anastomotic leaks, were not found to be statistically significant. Patients who experienced surgical complications started their chemotherapy later than patients who did not experience such complications (28.2 \pm 18.00 vs. $17.1 \pm 15.40, \mathrm{P}=0.002)$. In addition to these complications, the hospital stays of 26 patients in the laparoscopic group and 12 patients in the open surgery group were prolonged because they received their first postoperative adjuvant chemotherapy before they were discharged (Table 3 ).

We also investigated causes for patients not being able to complete their adjuvant chemotherapy. Five patients in the laparoscopic group and 3 patients in the open surgery group failed to complete their adjuvant chemotherapy due to nausea, diarrhea, dizziness, etc. $(\mathrm{P}=0.641)$. In addition, 4 patients in the laparoscopic group and 5 patients in the open surgery group discontinued their chemotherapy due to sepsis, pneumonia, neutropenia, and bowel obstruction $(\mathrm{P}=0.221)$. Eleven patients in the laparoscopic group and 10 patients in open surgery group voluntarily discontinued their adjuvant chemotherapy $(\mathrm{P}=0.232)$.

As a result of analyzing the relationship between clinical and pathological factors and the completion rate of adjuvant chemotherapy regardless of the surgical method, the completion rate
Table 3. Causes of long hospital stay

\begin{tabular}{lccc}
\hline Variable & LS & OS & P-value \\
\hline Wound infection & $3(3.3)$ & $7(12.5)$ & 0.043 \\
Anastomotic leak & $2(2.2)$ & $1(1.8)$ & 1.000 \\
Urinary complication & $7(7.6)$ & $1(1.8)$ & 0.156 \\
lleus & $13(14.3)$ & $11(19.6)$ & 0.507 \\
Chemotherapy & $26(28.6)$ & $12(21.4)$ & 0.483 \\
\hline
\end{tabular}

Values are presented as number (\%).

LS, laparoscopic surgery; OS, open surgery.

was statistically significantly higher for patients with ASA scores of 1 or 2 points $(P=0.004)$, for patients whose ages were less than 70 years $(\mathrm{P}=0.058)$, and for patients whose BMIs were equal to or more than $20(\mathrm{P}=0.051)$; however, the results were not statistically significant. In addition, patient's gender, stage of cancer, and types of insurance were not correlated to the completion rate of adjuvant chemotherapy (Table 4).

We also analyze results for each group, laparoscopic and open surgery. In the laparoscopic group, the completion rate of adjuvant chemotherapy was not correlated to patient's age $(\mathrm{P}=0.316)$ or BMI $(\mathrm{P}=0.231)$. On the other hand, 64 of 77 patients with ASA scores of 1 or 2 points and 7 of 13 patients with ASA scores 3 or 4 points completed their adjuvant chemotherapy. These results were statistically significant $(81.8 \%$ vs. $53.4 \% ; \mathrm{P}=0.035)$. In the open surgery group, the completion rate of adjuvant chemotherapy was higher for patients whose ages were less than 70 years $(\mathrm{P}=0.010)$, but it was not related to the ASA score with any statistical significance $(\mathrm{P}=0.066)$. Also, its relationship with the BMI was not statistically significant $(\mathrm{P}=0.115)$.

\section{DISCUSSION}

Over the last 10 years, laparoscopic surgery for colon cancer has drastically increased. Many factors have contributed to this increase. The dissemination and implantation of cancer cells at the port site can be successfully prevented during the surgical procedure. Also, many studies have reported on the short- and the long-term follow-up results of laparoscopic and open surgery, and those results have consistently shown the oncological outcome of laparoscopic surgery not to be inferior to outcome of open surgery. This is the major reason laparoscopic surgery is recognized as a safe surgical method nowadays. Moreover, advances in surgical techniques and development of various laparoscopic surgical instruments are other causes for this increase [6].

The stage of cancer, its histologic grade, lymphovascular invasion, and adjuvant chemotherapy have been reported to be factors that influence the survival rate of colon cancer patients $[7,8]$. Adjuvant chemotherapy has improved the survival rate of patients with various solid tumors. However, it is difficult to expect an improved survival rate due to adjuvant chemotherapy if the compliance with 
Volume 30, Number 6, 2014

Ann Coloproctol 2014;30(6):274-279

Table 4. Completion rate of chemotherapy according to the patient's clinico-pathologic characteristics

\begin{tabular}{|c|c|c|c|c|c|c|}
\hline Variable & LS & P-value & OS & P-value & Overall & P-value \\
\hline Age (yr) & & 0.316 & & 0.010 & & 0.058 \\
\hline$>70$ & 29/39 (74.3) & & 20/23 (87.0) & & 16/22 (72.2) & \\
\hline$\leq 70$ & $42 / 52(80.7)$ & & 18/33 (54,5) & & 16/29 (55.2) & \\
\hline Sex & & 0.489 & & 0.139 & & 0.230 \\
\hline Male & 45/57 (78.9) & & 30/41 (73.2) & & 75/98 (76.5) & \\
\hline Female & 26/34 (76.5) & & 8/15 (53.3) & & $34 / 49$ (69.4) & \\
\hline Stage & & 0.316 & & 0.090 & & 0.061 \\
\hline$\|$ & $36 / 48(75.0)$ & & $21 / 35(60.0)$ & & $57 / 83(68.7)$ & \\
\hline III & $35 / 43(81.4)$ & & 17/21 (81.0) & & 52/64 (81.2) & \\
\hline ASA score & & 0.035 & & 0.066 & & 0.004 \\
\hline 1,2 & 63/77 (81.8) & & $33 / 44(75.0)$ & & $96 / 121$ (79.3) & \\
\hline 3,4 & 7/13 (53.8) & & $5 / 11$ (45.5) & & 12/24 (50.0) & \\
\hline Health insurance & & 0.438 & & 0.085 & & 0.079 \\
\hline $\mathrm{NHI}$ & 64/83 (77.1) & & $32 / 50(64.0)$ & & 96/133 (72.2) & \\
\hline Medicaid & 7/8 (87.5) & & 6/6 (100) & & 13/14 (92.9) & \\
\hline Chemotherapy & & 0.489 & & 0.078 & & 0.193 \\
\hline Intravenous & $33 / 43(76.7)$ & & 15/18 (83.3) & & 48/61 (78.7) & \\
\hline Oral & 38/48 (79.2) & & 23/38 (60.5) & & 61/86 (70.9) & \\
\hline BMI $\left(\mathrm{kg} / \mathrm{m}^{2}\right)$ & & 0.231 & & 0.115 & & 0.051 \\
\hline$\geq 20$ & 8/12 (66.7) & & 4/9 (44.4) & & 12/21 (57.1) & \\
\hline$<20$ & 62/77 (80.5) & & $33 / 46(71.7)$ & & 95/123 (77.2) & \\
\hline Operation type & & 0.220 & & 1.000 & & 0.451 \\
\hline Elective & 71/90 (78.8) & & 37/55 (67.3) & & $108 / 145(74.5)$ & \\
\hline Emergency & $0 / 1(0)$ & & 1/1 (100) & & $1 / 2(50.0)$ & \\
\hline Postoperative complication & & 0.237 & & 0.558 & & 0.246 \\
\hline$x$ & $60 / 70(85.7)$ & & $31 / 41(75.6)$ & & $91 / 111(82.0)$ & \\
\hline 0 & 16/21 (76.2) & & $11 / 15(73.3)$ & & 27/36 (75.0) & \\
\hline
\end{tabular}

Values are presented as number/total number (\%).

LS, laparoscopic surgery; OS, open surgery; ASA, American Society of Anesthesiologists; BMI, body mass index; NHI, National Health Insurance.

adjuvant chemotherapy in patients is poor due to its side effects [9]. Many studies have reported that age, gender, postoperative complications, the severity of the side effects of chemotherapy, and patients' socioeconomic status are factors that have an influence on the completion of adjuvant chemotherapy [10-13].

In this study, the hospital stay was shorter, and the adjuvant chemotherapy started earlier in the laparoscopic group; these differences were statistically significant. In addition, the completion rate of adjuvant chemotherapy and the survival rate were higher. Many other studies have also reported mostly shorter hospital stays in laparoscopic groups $[14,15]$, which is consistent with the results of this study $(\mathrm{P}=0.001)$. However, the hospital stay in this study was longer than it was in previous studies. This seems to be due to the facts that many patients received their first postoperative adjuvant chemotherapy before their discharge, that some pa- tients experienced postoperative complications, and that some patients resided location far from the hospital. Therefore, the average length of the hospital stay was prolonged in this study.

Some studies have reported that delayed adjuvant chemotherapy leads to a lower overall survival rate $[16,17]$. In this study, the chemotherapy was started, on average, 17.7 days after surgery in laparoscopic group, which was earlier than the open surgery group, the difference being statistically significant $(\mathrm{P}=0.044)$. This is thought to have been due to patients in the laparoscopic group experiencing less pain and having a lower incidence of surgical wound infection, one of the complications.

The laparoscopic and the open surgery groups showed $78 \%$ and $67.8 \%$ completion rates of adjuvant chemotherapy, respectively. The result was higher in the laparoscopic group, but this results had no statistical significance $(\mathrm{P}=0.121)$. The completion rate of 
adjuvant chemotherapy was, with statistical significance, related to the group with the shorter hospital stay after surgery $(\mathrm{P}=0.020)$ and to the group with an earlier initiation of adjuvant chemotherapy $(P=0.029)$. Because the laparoscopic surgery group has the advantages of shorter hospital stay and earlier initiation of adjuvant chemotherapy, the choice of laparoscopic surgery is thought to have an indirect effect on the completion of adjuvant chemotherapy, but further studies with more patients are required.

In this study, factors, including BMI and ASA score, had an influence on the completion of adjuvant chemotherapy. Frank et al. [14]. reported that BMI affected the survival rate of patients. They investigated patients who received 5-FU-based adjuvant chemotherapy and found that the mortality was higher in obese and underweight patients than it was in patients of normal weight group and that the disease-free survival was shorter in the underweight group.

This study found the completion rate of adjuvant chemotherapy to be $57.1 \%(12 / 21)$ for patients who were underweight, which was lower than the $77.2 \%$ (95/123) for patients of normal weight or patients who were overweight $(\mathrm{P}=0.051)$. However, our sample size was very small; thus, further studies should include more patients in order to give statistical significance to this result.

Previous studies reported that the completion rate of adjuvant chemotherapy was lower in older patients [12, 13]. In this study, the patients whose ages were less than 70 years showed a higher completion rate than the patients whose ages were over 70. Especially, in the open surgery group, the completion rate of adjuvant chemotherapy was significantly different, depending on age $(\mathrm{P}=$ 0.002). On the other hand, no difference in the completion rates was found in the laparoscopic group. Therefore, we can conclude that laparoscopic surgery leads to an improvement in the completion rate of adjuvant chemotherapy, even in elderly patients.

In this study, the ASA score was also, with statistical significance, related to the completion rate of adjuvant chemotherapy. In the laparoscopic group, the completion rate of adjuvant chemotherapy was higher in patients with ASA scores of 1 or 2 than it was in patients with ASA scores of 3 or 4; however, no such difference was observed in the open surgery group. These results show that the preoperative physical status of patients is associated with the completion rate of adjuvant chemotherapy and, especially in the laparoscopic group, underlying diseases and low physical activity levels are thought to be factors that may decrease the completion rate.

Sharon et al. [10]. studied the completion rate of adjuvant chemotherapy in 3,193 patients with stage III colon cancer. They reported no statistically significant difference based on gender, even though it was higher for male patients, which is consistent with the observation in this study. In our case, statistical significance may not have been proven due to the small sample size. Cathy et al. [17]. reported that patients who were supported by the medical aid program usually started their adjuvant chemotherapy late, and its completion rate was low. However, in this study, no statistically significant difference was noted between patients covered by Ko- rea's National Health Insurance and patients covered by the medical aid program. These differences in results are thought to be due to differences in the medical insurance systems between Korea and the USA.

According to many previous studies, no statistically significant differences in the overall survival rate of patients with colorectal cancer between laparoscopic and open surgery groups were found $[18,19]$. However, in this study, the laparoscopic group showed a $96.7 \%$ (88 of 91) survival rate compared to $85.7 \%$ (48 of 56 ) in the open surgery group $(\mathrm{P}=0.007)$. However, many patients with a high ASA score were included in the open surgery group, and accompanying diseases were not analyzed in this study. In addition, the follow-up term was short. Therefore, a long-term follow-up and survival analysis are required to give statistical significance to this result.

In conclusion, the completion rate of adjuvant chemotherapy was lower in patients with a low ASA score in this study. Also, the hospital stay was shorter and adjuvant chemotherapy started earlier in the patients who underwent laparoscopic surgery than in the patients who underwent open surgery for colon cancer. We conclude that laparoscopic surgery contributes to improved compliance with adjuvant chemotherapy.

\section{CONFLICT OF INTEREST}

No potential conflict of interest relevant to this article was reported.

\section{REFERENCES}

1. Jung KW, Won YJ, Kong HJ, Oh CM, Lee DH, Lee JS. Cancer statistics in Korea: incidence, mortality, survival, and prevalence in 2011. Cancer Res Treat 2014;46:109-23.

2. Gondos A, Bray F, Hakulinen T, Brenner H; EUNICE Survival Working Group. Trends in cancer survival in 11 European populations from 1990 to 2009: a model-based analysis. Ann Oncol 2009;20:564-73.

3. Green BL, Marshall HC, Collinson F, Quirke P, Guillou P, Jayne DG, et al. Long-term follow-up of the Medical Research Council CLASICC trial of conventional versus laparoscopically assisted resection in colorectal cancer. Br J Surg 2013;100:75-82.

4. Fleshman J, Sargent DJ, Green E, Anvari M, Stryker SJ, Beart RW Jr, et al. Laparoscopic colectomy for cancer is not inferior to open surgery based on 5-year data from the COST Study Group trial. Ann Surg 2007;246:655-62.

5. Hazebroek EJ; Color Study Group. COLOR: a randomized clinical trial comparing laparoscopic and open resection for colon cancer. SurgEndosc 2002;16:949-53.

6. Bagshaw PF, Allardyce RA, Frampton CM, Frizelle FA, Hewett PJ, McMurrick PJ, et al. Long-term outcomes of the australasian randomized clinical trial comparing laparoscopic and conventional open surgical treatments for colon cancer: the Australasian Lapa- 
roscopic Colon Cancer Study trial. Ann Surg 2012;256:915-9.

7. Yun HR, Kim HC, Yun SH, Lee WY. Adjuvant chemotherapy increase survival and decrease recurrence in stage IIA colon cancer. Hepatogastroenterology 2012;59:2466-71.

8. Benson AB 3rd, Bekaii-Saab T, Chan E, Chen YJ, Choti MA, Cooper HS, et al. Localized colon cancer, version 3.2013: featured updates to the NCCN Guidelines. J NatlComprCancNetw 2013;11: 519-28.

9. Morris M, Platell C, Fritschi L, Iacopetta B. Failure to complete adjuvant chemotherapy is associated with adverse survival in stage III colon cancer patients. Br J Cancer 2007;96:701-7.

10. Dobie SA, Baldwin LM, Dominitz JA, Matthews B, Billingsley K, Barlow W. Completion of therapy by Medicare patients with stage III colon cancer. J Natl Cancer Inst 2006;98:610-9.

11. Sinicrope FA, Foster NR, Yothers G, Benson A, Seitz JF, Labianca $\mathrm{R}$, et al. Body mass index at diagnosis and survival among colon cancer patients enrolled in clinical trials of adjuvant chemotherapy. Cancer 2013;119:1528-36.

12. Hu CY, Delclos GL, Chan W, Du XL. Assessing the initiation and completion of adjuvant chemotherapy in a large nationwide and population-based cohort of elderly patients with stage-III colon cancer. Med Oncol 2011;28:1062-74.

13. van der Geest LG, Portielje JE, Wouters MW, Weijl NI, Tanis BC, Tollenaar RA, et al. Complicated postoperative recovery increases omission, delay and discontinuation of adjuvant chemotherapy in patients with Stage III colon cancer. Colorectal Dis 2013;15:e58291.

14. Clinical Outcomes of Surgical Therapy Study Group. A comparison of laparoscopically assisted and open colectomy for colon cancer. N Engl J Med 2004;350:2050-9.

15. Lee JE, Joh YG, Yoo SH, Jeong GY, Kim SH, Chung CS, et al. Longterm outcomes of laparoscopic surgery for colorectal cancer. J Korean SocColoproctol 2011;27:64-70.

16. Bayraktar UD, Chen E, Bayraktar S, Sands LR, Marchetti F, Montero AJ, et al. Does delay of adjuvant chemotherapy impact survival in patients with resected stage II and III colon adenocarcinoma? Cancer 2011;117:2364-70.

17. Czaykowski PM, Gill S, Kennecke HF, Gordon VL, Turner D. Adjuvant chemotherapy for stage III colon cancer: does timing matter? Dis Colon Rectum 2011;54:1082-9.

18. Bradley CJ, Given CW, Dahman B, Fitzgerald TL. Adjuvant chemotherapy after resection in elderly Medicare and Medicaid patients with colon cancer. Arch Intern Med 2008;168:521-9.

19. Colon Cancer Laparoscopic or Open Resection Study Group, Buunen M, Veldkamp R, Hop WC, Kuhry E, Jeekel J, et al. Survival after laparoscopic surgery versus open surgery for colon cancer: long-term outcome of a randomised clinical trial. Lancet Oncol 2009;10:44-52. 